\title{
Uncertainty Quantification for Stochastic Nonlinear Systems using Perron-Frobenius Operator and Karhunen-Loève Expansion
}

\author{
Parikshit Dutta ${ }^{\dagger}$, Abhishek Halder and Raktim Bhattacharya
}

\begin{abstract}
In this paper, a methodology for propagation of uncertainty in stochastic nonlinear dynamical systems is investigated. The process noise is approximated using KarhunenLoève (KL) expansion. Perron-Frobenius (PF) operator is used to predict the evolution of uncertainty. A multivariate Kolmogorov-Smirnov test is used to verify the proposed framework. The method is applied to predict uncertainty evolution in a Duffing oscillator and a Vanderpol's oscillator. It is observed that the solution of the approximated stochastic dynamics converges to the true solution in distribution. Finally, the proposed methodology is combined with Bayesian inference to estimate states of a nonlinear dynamical system, and its performance is compared with particle filter. The proposed estimator was found to be computationally superior than the particle filter.
\end{abstract}

\section{INTRODUCTION}

Consider the stochastic nonlinear system

$$
\begin{aligned}
d x(t) & =f(x(t), \delta) d t+g_{w}(x(t), \delta) d w(t), \\
y(t) & =h(x(t), \delta)+g_{\eta}(x(t), \delta) \eta(t),
\end{aligned}
$$

where $x(t) \in \mathbb{R}^{n}$ and $y(t) \in \mathbb{R}^{m}$ are the state and measurement vectors at time $t, \delta \in \mathbb{R}^{p}$ is the parameter vector, and $w(t) \in \mathbb{R}^{q}, \eta(t) \in \mathbb{R}^{l}$ are mutually independent Wiener processes denoting process and measurement noise, respectively. The functions $f(.,),. h(.,$.$) represent the dy-$ namics and measurement model while the function-valued matrices $g_{w}(.,),. g_{\eta}(.,$.$) denote respective noise coupling.$

If $f(.,$.$) and h(.,$.$) are linear with constant noise cou-$ pling, the uncertainty quantification reduces to updating the mean and covariance propagation equations, and the associated estimation problem is solved by the well-known Kalman filter [1]. For general nonlinear systems, if the uncertainty in the initial conditions $\left(x_{0}\right)$ and parameters $(\delta)$ are prescribed through the joint probability density function (PDF) $\rho_{0}(z), z:=\left[\begin{array}{ll}x & \delta\end{array}\right]^{T} \in \mathbb{R}^{n+p}$, then the propagation of uncertainty through (1) and (2) requires solving the FokkerPlanck equation (or Kolmogorov forward equation)

$$
\frac{\partial \rho}{\partial t}=-\sum_{i=1}^{n} \frac{\partial}{\partial x_{i}}\left(\rho f_{i}\right)+\sum_{i=1}^{n} \sum_{j=1}^{n} \frac{\partial^{2}}{\partial x_{i} \partial x_{j}}\left(\left(g_{w} Q g_{w}^{T}\right)_{i j} \rho\right)
$$

$\dagger$ Corresponding author

P. Dutta is with INRIA Rhône-Alpes, 655 avenue de l'Europe, 38330 Montbonnot, France, parikshit.duttaeinria.fr.

A. Halder and R. Bhattacharya are with the Department of Aerospace Engineering, Texas A\&M University, College Station, TX 77843, USA, \{ahalder, raktim\}@tamu.edu.

This research was partially supported by NSF award \# 1016299 with D. Helen Gill as the Program Manager. to compute $\rho(z, t)$ subject to $\rho(z, 0)=\rho_{0}(z)$, where $\mathbb{E}\left[d w_{i}\right]=0$ and $\mathbb{E}\left[d w_{i} d w_{j}\right]=Q_{i j} \geqslant 0, \forall i, j=1,2, \ldots, q$.

Solving the parabolic partial differential equation (PDE) (3), second order in space and first order in time, is computationally hard [2] and the approximation algorithms often suffer from the 'curse of dimensionality' [3]. However, in the absence of process noise, (3) reduces to the Liouville equation associated with the Perron-Frobenius operator [4] that describes the spatio-temporal drift of $\rho(z, t)$ in the presence of parametric and initial condition uncertainty. Being a first order quasi-linear PDE, Liouville equation can be solved exactly [5] using the method of characteristics (MOC) [6] and unlike brute-force Monte-Carlo, such a computation does not compromise with runtime complexity [7], [8]. In nonlinear estimation setting, the prior PDF obtained through this propagation, can be used to compute the posterior PDF via Bayesian update. The resulting nonlinear filter has been shown [9] to outperform particle filter and bootstrap filter.

Instead of nonparametric propagation of the PDF, one may use a polynomial chaos [10] expansion to quantify uncertainty, where a set of deterministic ordinary differential equations (ODEs) is derived using either Galerkin projection [11] or stochastic collocation [12]. There are three bottlenecks with this parametric approach. First, it's difficult to derive the deterministic ODEs for general nonlinearities [13]. Second, the finite dimensional approximation of the probability space degrades the accuracy of long-term statistics. Third, the deterministic set of ODEs requires solution in a higher dimensional state space than the original, making the computation prohibitively expensive for large scale systems. Other parametric methods like moment closure lack accuracy due to approximation of the PDF using first few moments.

The objective of this paper is to introduce a mixed parametric-nonparametric approach for propagating uncertainty in the presence of process noise. Starting from the Langevin equation [14], by $N_{t}$-term Karhunen-Loève expansion [15] of the process noise, we derive a deterministic ODE corresponding to the Itô stochastic differential equation (SDE) (1). Then the Perron-Frobenius operator based formulation is used to exactly solve the approximated SDE in distributional sense. A detailed verification procedure is illustrated to assess the quality of the $N_{t}$-term approximation. There are two main contributions:

1) We show that for the nonlinear stochastic system (1) and (2), instead of solving (3), $\rho(z, t)$ can be computed 
along the trajectories of an approximately equivalent deterministic dynamics over the extended state space. This enables us to harness the computational benefits of the Perron-Frobenius operator based formulation even in the presence of process noise.

2) We present a method for probabilistic verification of the approximated solution based on multivariate hypothesis testing. Consequently, for a fixed number of terms in the Karhunen-Loève expansion, we can compute the significance level of the finite-sample computation. Conversely, if a desired level of verification is specified, the number of terms for approximating the process noise can be computed.

This paper is structured as follows. In section II, we show that the nonparametric propagation of the joint PDF $\rho(z, t)$ can be achieved by solving the Liouville equation associated with the Perron-Frobenius operator, subject to the deterministic dynamics obtained from the Langevin equation by approximating the process noise through KarhunenLoève expansion. Some examples are given in section III to illustrate the proposed formulation. Section IV describes the verification framework followed by applications to nonlinear filtering in section V. Section VI concludes the paper.

\section{Methodology}

We consider the Langevin equation

$$
\frac{d}{d t} x(t)=f(x(t), \delta)+\xi(t)
$$

associated with the Itô SDE (1) for purely additive Gaussian white noise $\xi(t)$ with autocorrelation $\sigma^{2} \mathbf{I}$. In the sequel, we first describe the method for uncertainty propagation via Perron-Frobenius operator followed by Karhunen-Loève expansion of the process noise. Next, we combine these two to compute the evolution of $\rho(z, t)$ corresponding to (4).

\section{A. Perron-Frobenius Operator}

Definition 1: (Continuous-time Perron-Frobenius operator) Let $\mathcal{X}$ be endowed with a measure space $(\mathcal{X}, \mathcal{B}, \mu)$ and that all transformations $f_{t}$ of a semi-dynamical system [4] $\left\{f_{t}\right\}_{t \geqslant 0}$ are nonsingular (i.e. $\mu\left(f_{t}^{-1}(B)\right)=0, \forall B \in \mathcal{B}$ such that $\mu(B)=0$ ), then for each $t \geqslant 0$, the PerronFrobenius operator $\mathcal{P}_{t}: L_{1}(\mathcal{X}) \mapsto L_{1}(\mathcal{X})$, corresponding to the dynamics $f_{t}$, is uniquely defined as

$\int_{B} \mathcal{P}_{t} \rho(x) \mu(d x)=\int_{f_{t}^{-1}(B)} \rho(x) \mu(d x), \quad$ for $B \in \mathcal{B}$.

The following properties of the Perron-Frobenius operator are important in our context, $\forall \rho, \rho_{1}, \rho_{2} \in L_{1}, \quad \alpha, \beta \in \mathbb{R}$.

1) (Linearity) $\mathcal{P}_{t}\left(\alpha \rho_{1}+\beta \rho_{2}\right)=\alpha \mathcal{P}_{t} \rho_{1}+\beta \mathcal{P}_{t} \rho_{2}$,

2) (Non-negativity) $\mathcal{P}_{t} \rho \geqslant 0$, provided $\rho \geqslant 0$;

3) (Conservation) $\int_{\mathcal{X}} \mathcal{P}_{t} \rho(x) \mu(d x)=\int_{\mathcal{X}} \rho(x) \mu(d x)$, The above properties guarantee that the operator $\mathcal{P}_{t}$ : $L_{1}(\mathcal{X}) \mapsto L_{1}(\mathcal{X})$, is a Markov operator. In particular, property 3 assures that during Markov evolution, PDF respects the normality condition $\int_{\mathcal{X}} \rho(x) \mu(d x)=1$, where $\mu($.$) is$ the Lebesgue measure.
One can show [4] that the spatio-temporal evolution of the joint PDF via Perron-Frobenius operator (5), subject to the dynamics (4) with $\xi(t)=0$, satisfies the Liouville PDE

$$
\frac{\partial \rho(x, t)}{\partial t}=-\sum_{i=1}^{n} \frac{\partial}{\partial x_{i}}\left(\rho(x, t) f_{i}\right)
$$

which is simply (3) except the diffusion term. Being a first order PDE, (6) can be exactly solved using MOC along the characteristic curves. It can be shown [5] that the characteristic curves for (6) are nothing but the trajectories of the extended dynamics $\dot{z}=\left[\begin{array}{ll}f(z) & \mathbf{0}_{p \times 1}\end{array}\right]^{T}$. The computational performance of this formalism is known [7] to be superior than Monte-Carlo. However, in its original form, only initial condition and parametric uncertainties can be accounted for.

\section{B. Karhunen-Loève Expansion of Process Noise}

Karhunen-Loève (KL) expansion was derived independently by many researchers [15], [16] to represent a stochastic process $Y(\omega, t)$ as a random linear combination of a set of orthonormal deterministic $L_{2}$ functions $\left\{e_{i}(t)\right\}_{i=1}^{\infty}$, i.e.

$$
Y(\omega, t)=\sum_{i=1}^{\infty} Z_{i}(\omega) e_{i}(t)
$$

The idea is similar to the Fourier series expansion, where a deterministic linear combination of orthonormal $L_{2}$ functions is used. It's worth mentioning that in (7), one can replace $t$ by $x \in \mathbb{R}^{n}$, to have $\mathrm{KL}$ expansion of a random field [17]. Further, if we write $Z_{i}(\omega)=\sqrt{\lambda_{i}} \zeta_{i}(\omega)$, where $\lambda_{i} \in \mathbb{R}^{+}$and $\left\{\zeta_{i}(\omega)\right\}_{i=1}^{\infty}$ is a sequence of random variables to be determined, then $\left\{\lambda_{i}\right\}_{i=1}^{\infty}$ and $\left\{e_{i}(t)\right\}_{i=1}^{\infty}$ can be interpreted as the eigenvalues and eigenfunctions of the covariance function [18] $C\left(t_{1}, t_{2}\right):=$ $\operatorname{cov}\left(Y\left(\omega, t_{1}\right)-\mathbb{E}\left[Y\left(\omega, t_{1}\right)\right], Y\left(\omega, t_{2}\right)-\mathbb{E}\left[Y\left(\omega, t_{2}\right)\right]\right)$, that admits a spectral decomposition [19] of the form $C\left(t_{1}, t_{2}\right)=\sum_{i=1}^{\infty} \lambda_{i} e_{i}\left(t_{1}\right) e_{i}\left(t_{2}\right)$. Since the covariance function is bounded, symmetric and positive-definite, the eigenvalue problem can be cast as a homogeneous Fredholm integral equation of second kind, given by, $\int_{\mathcal{D}_{t}} C\left(t_{1}, t_{2}\right) e_{i}\left(t_{1}\right) d t_{1}=\lambda_{i} e_{i}\left(t_{2}\right)$. Given the covariance function of a stochastic process, the eigenvalue-eigenfunction set can be found by solving Fredholm equation, and the resulting expansion (7) converges to $Y(\omega, t)$ in mean-square sense [20]. In particular, the following result is useful for our purpose.

Theorem 1: (KL expansion of Wiener process) (p. 548, [20]) For Wiener process, the eigenvalues and eigenfunctions of the covariance function $C\left(t_{1}, t_{2}\right)=\left(t_{1} \wedge t_{2}\right), t_{1}, t_{2} \in$ $[0, T] \times[0, T]$, is given by

$\lambda_{i}=\frac{4 T}{\pi^{2}(2 i-1)^{2}}, \quad e_{i}(t)=\sqrt{2} \sin \left(\left(i-\frac{1}{2}\right) \frac{\pi t}{T}\right)$, 
for $i=1,2, \ldots$, and hence the KL expansion (7) for $w(t)$, is of the form

$$
w(\omega, t) \stackrel{\text { m.s. }}{=} \sqrt{2} \sum_{i=1}^{\infty} \zeta_{i}(\omega) \frac{\sin \left(\left(i-\frac{1}{2}\right) \frac{\pi t}{T}\right)}{\left(i-\frac{1}{2}\right) \frac{\pi}{T}},
$$

where $\zeta_{i}(\omega)$ are i.i.d. samples drawn from $\mathcal{N}\left(0, \sigma^{2}\right)$.

Corollary 2: (KL expansion of Gaussian white noise) Since $d w(t)=\xi(t) d t$, the KL expansion for Gaussian white noise $\xi(t)$ can be obtained by taking the derivative of (9) with respect to $t$, i.e.

$$
\xi(t) \stackrel{\text { m.s. }}{=} \sqrt{2} \sum_{i=1}^{\infty} \zeta_{i}(\omega) \cos \left(\left(i-\frac{1}{2}\right) \frac{\pi t}{T}\right),
$$

where the i.i.d. random variables $\zeta_{i}(\omega) \sim \mathcal{N}\left(0, \sigma^{2}\right)$. By substituting (10) in (4), we obtain a deterministic approximation of the stochastic dynamics (1). More generally, expansions of this nature can be derived in closed form, when the stochastic forcing in (1) can be written as a functional of Wiener process (e.g. Brownian bridge [21]).

\section{KLPF Formulation}

Let us consider the dynamical system given in (4) where $x \in \mathbb{R}^{n}$ are the states, and $z \in \mathcal{D}_{z}$ is the vector of state and parametric uncertainty, having initial $\operatorname{PDF} \rho_{0}(z)$. Without loss of generality, it is assumed, that the system has only initial state uncertainty, hence $z(t=0):=x(t=0)$. The stochastic forcing term $\xi(t)$ in (4) is approximated using KL expansion having finite number of terms $\left(N_{t}\right)$. In the present case, where $\xi(t)$ is a Gaussian white noise, the approximation reduces to, $\xi(t)=\sqrt{2} \sum_{i=1}^{N_{t}} \zeta_{i}(\omega) \cos \left(\left(i-\frac{1}{2}\right) \frac{\pi t}{T}\right)$, where $t \in[0, T]$. After substituting this expression in (4), we get the following differential equation,

$$
\dot{x}(t)=f(x(t))+\sqrt{2} \sum_{i=1}^{N_{t}} \zeta_{i}(\omega) \cos \left(\left(i-\frac{1}{2}\right) \frac{\pi t}{T}\right),
$$

Using the methodology given in [5], MOC is applied to (6), and an augmented dynamical system is formed, with states $\left[x_{1}(t), \ldots, x_{n}(t), \rho(x(t))\right]^{T}$, where $\rho(x(t))$ is the probability density of the states at time $t$, and $x(t)=$ $\left[x_{1}(t), \ldots, x_{n}(t)\right]^{T}$. The augmented system is given by,

$$
\begin{aligned}
\dot{x}(t) & =f(x(t))+\sqrt{2} \sum_{i=1}^{N_{t}} \zeta_{i}(\omega) \cos \left(\frac{\pi t(2 i-1)}{2 T}\right), \\
\dot{\rho}(x(t)) & =-\operatorname{div} f(x(t)) \rho(x(t)),
\end{aligned}
$$

where $\operatorname{div} f(x(t))=\sum_{i=1}^{n} \frac{\partial f(x(t))}{\partial x_{i}(t)}$. Equation (12) can be solved to get the value of $\rho(x(t))$ along the characteristic curves of (6). Detailed discussion of the solution methodology has been omitted here, and can be found in [8], [9].

\section{ILLUSTRATIVE EXAMPLES}

The proposed methodology is applied to a Vanderpol's oscillator and a Duffing oscillator. The augmented dynamical system for the Vanderpol's oscillator is given by,

$$
\begin{aligned}
\dot{x}_{1}(t) & =x_{2}(t) \\
\dot{x}_{2}(t) & =\left(1-x_{1}^{2}(t)\right) x_{2}(t)-x_{1}(t)+\xi(t) \\
\dot{\rho}(x(t)) & =-\left(1-x_{1}^{2}(t)\right) \rho(x(t))
\end{aligned}
$$

and for the Duffing oscillator, is given by,

$$
\begin{aligned}
\dot{x}_{1}(t) & =x_{2}(t) \\
\dot{x}_{2}(t) & =10 x_{1}(t)-30 x_{1}^{3}(t)-10 x_{2}(t)+\xi(t) \\
\dot{\rho}(x(t)) & =10 \rho(x(t))
\end{aligned}
$$

with $\xi(t)$ having autocorrelation $2 \pi \mathbf{I}$. The initial state uncertainty, has a PDF $\rho_{0}(z) \sim \mathcal{N}\left([0,0]^{T}, \operatorname{diag}(1,1)\right)$ for both the systems. Next, the initial PDF, $\rho_{0}(z)$ is sampled, with sample size of $N=5000$. For the Vanderpol's oscillator, final time $T$ is $1 \mathrm{~s}$, for the Duffing oscillator $T=3 \mathrm{~s}$. Number of terms in the KL expansion is fixed to $N_{t}=7$. Figure (1) shows the evolution of probability densities with time for the two oscillators. The density value $\rho(x(t))$ is color coded with red representing high density value and blue representing low. It is observed that, for the Vanderpol's oscillator the probability mass accumulates along the limit cycle and for the Duffing oscillator, we get a bimodal PDF at final time. This is in agreement with the physical intuition of behavior of these systems.

\section{VERIFICATION OF SOLUTION}

In the proposed methodology, the process noise in (4) has been approximated using finite number of terms in KL expansion. Due to this fact, the solution obtained after propagation is not error-free. It is well known that, KL expansion converges in mean square sense, to the underlying stochastic process as $N_{t} \rightarrow \infty$. But, same argument cannot be extended regarding the convergence of states $x(t)$ of the dynamical system in (11). To obtain a meaningful solution, there should be some notion of convergence of the states in (11) to states in (4). Hence, verification of the obtained solution is important in this scenario. In the present work, we have verified that, given the finite term KL expansion of $\xi(t)$, the solution of the approximated dynamics in (11) converge to the true solution in distribution. We have used the Kolmogorov-Smirnov (KS) test to verify the solution of approximated dynamics, as detailed below.

The Kolmogorov-Smirnov test [22] is a statistical test used to compare a sample with a reference probability distribution. It quantifies a distance $D_{N}$, between reference cumulative distribution function (CDF), $F(x)$, and empirical CDF of the sample being tested, $F_{N}(x)$, which is given by $D_{N}=$ $\sup _{x}\left|F_{N}(x)-F(x)\right|$. Here, $N$ refers to sample size of the given sample. The null hypothesis is that the sample comes from the reference distribution. Given a significance level $\alpha$, the null hypothesis is accepted if

$$
\sqrt{N} D_{N} \leq K_{\alpha}, \text { where, } \operatorname{Pr}\left(K \leq K_{\alpha}\right)=1-\alpha .
$$



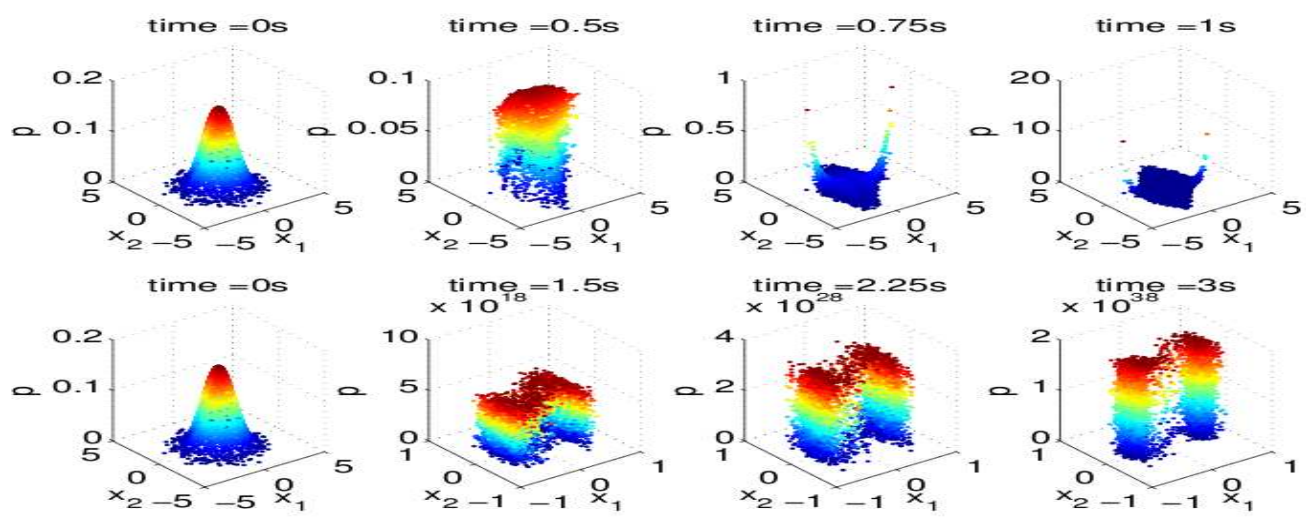

Fig. 1. Uncertainty Propagation for Vanderpol's Oscillator (13) in top row, and Duffing Oscillator (14) in bottom row.

Here, $K$ is a random variable that follows Kolmogorov distribution, with CDF

$$
\operatorname{Pr}(K \leq x)=\frac{\sqrt{2 \pi}}{x} \sum_{i=1}^{\infty} e^{-\frac{(2 i-1)^{2} \pi^{2}}{8 x^{2}}} .
$$

If the null hypothesis is accepted, then the KS test is passed and it can be concluded that the given sample comes from the reference PDF.

Due to the fact that $N<\infty, D_{N}$ is a random variable, as each sample will give us different $D_{N}$ values. The empirical distribution of $\sqrt{N} D_{N}$ is given by, $F_{N_{s}}(x)=$ $1 / N_{s}\left(\sum_{i=1}^{N_{s}} \mathcal{I}_{x<\sqrt{N} D_{N}^{i}}\right)$, where $N_{s}$ is the number of samples and $D_{N}^{i}$ is the $D_{N}$ value for $i^{t h}$ sample. The DvoretzkyKiefer-Wolfowitz inequality [23] characterizes the rate-ofconvergence of $F_{N_{s}}(x)$ as

$\operatorname{Pr}\left(\sup _{x \in \mathbb{R}}\left|F_{N_{s}}(x)-F_{K}(x)\right|>\varepsilon\right) \leq 2 e^{-2 N_{s} \varepsilon^{2}}, \forall \varepsilon>0$,

where $F_{K}(x)$ is the Kolmogorov CDF in (16). Hence as $N_{s} \rightarrow \infty$ the test statistic $\sqrt{N} D_{N}$ exponentially converges in distribution to a Kolmogorov random variable with rate $2 e^{-2 N_{s} \varepsilon^{2}}$.

In the present case, the analytical representation of PDF at time $t=T$ is not known. Hence to verify our solution, we back-propagate the sample obtained after propagation of (11) at final time $T$ to time $t=0$ using the original dynamics in (4); and check if the back-propagated sample belongs to the initial PDF $\rho_{0}(z)$.

Let the elements of the sample; sampled from the initial PDF be $x_{0, i}, i=1, \ldots, N$, and elements after propagation of (11) be $x_{T, i}$. Let the back-propagated sample obtained by propagating (4) from $[T, 0]$ be $\hat{x}_{0, i}$. Here, we claim that, $\hat{x}_{0, i}$ is a sample from the initial PDF. Hence, using the KS test, the sample $\hat{x}_{0, i}$, is compared with the initial PDF, for statistical significance. Here, we have used a multivariable KS test, which is similar to the one presented in [22]. The verification methodology is described in algorithm 1.

We apply the proposed verification methodology to the Vanderpol's oscillator given in (13). The initial state uncertainty has the PDF $\rho_{0}(z) \sim \mathcal{N}([0,0], \operatorname{diag}(1,1))$. We obtain $N_{s}=100$ samples, each of sample size $N=500$ from $\rho_{0}(z)$. The number of terms in the KL expansion is fixed to $N_{t}=21$. At first, we pick a sample from the 100 available

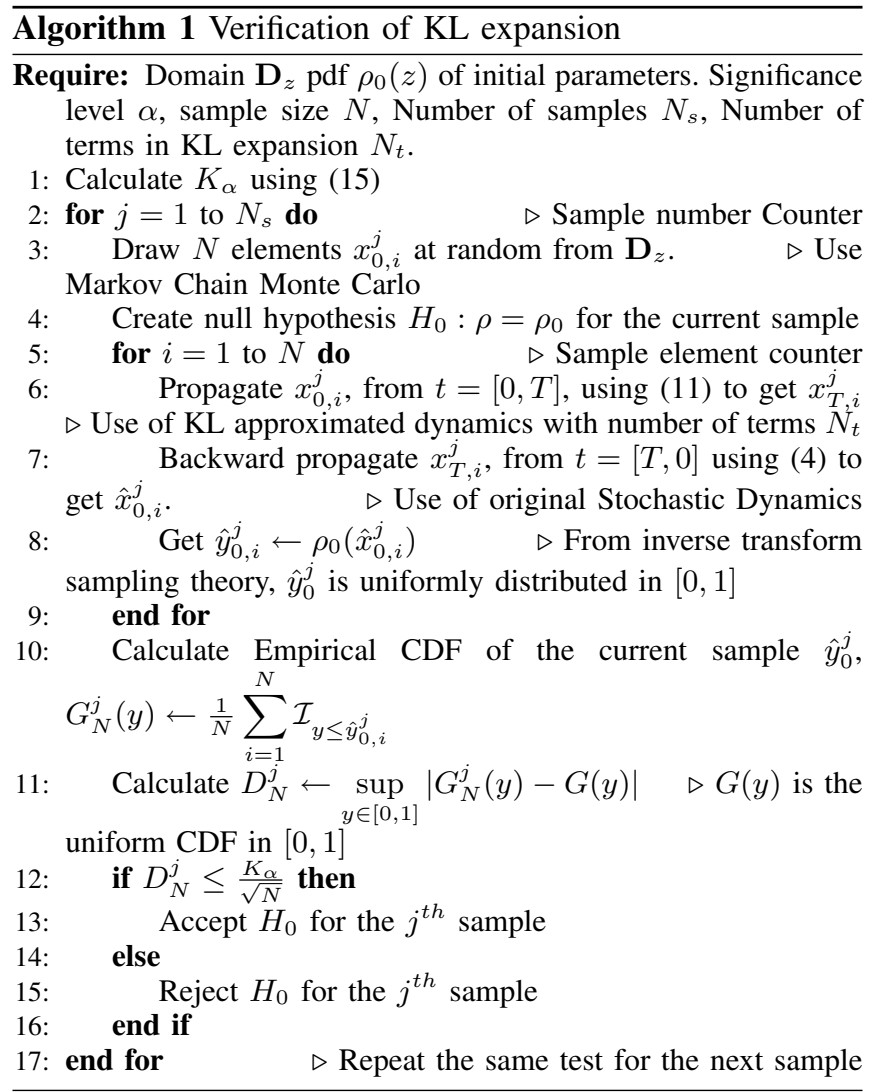

samples and apply the verification methodology. Figure (2) shows plot for the location of elements initially $\left(x_{0}\right)$ and after back-propagation $\left(\hat{x}_{0}\right)$, for the particular sample. It is observed that the back-propagated sample, is clustered around the origin and sparseness increases as we move away. This is in agreement with the physical intuition of random samples drawn from $\rho_{0}(z)$ which is a standard normal distribution.

Using the methodology in [22] we obtain uniformly distributed sample $\hat{y}_{0}=F\left(\hat{x}_{0}\right)$ using inverse transform sampling theory [25]. Figure (2) shows the plot of empirical $\mathrm{CDF}$ of $\hat{y}_{0}^{j}$ and the uniform CDF for the randomly selected sample. It can be seen that, visually the CDFs are close to each other. Hence, the value of $D_{N}$ for the particular sample is expected to be less. 


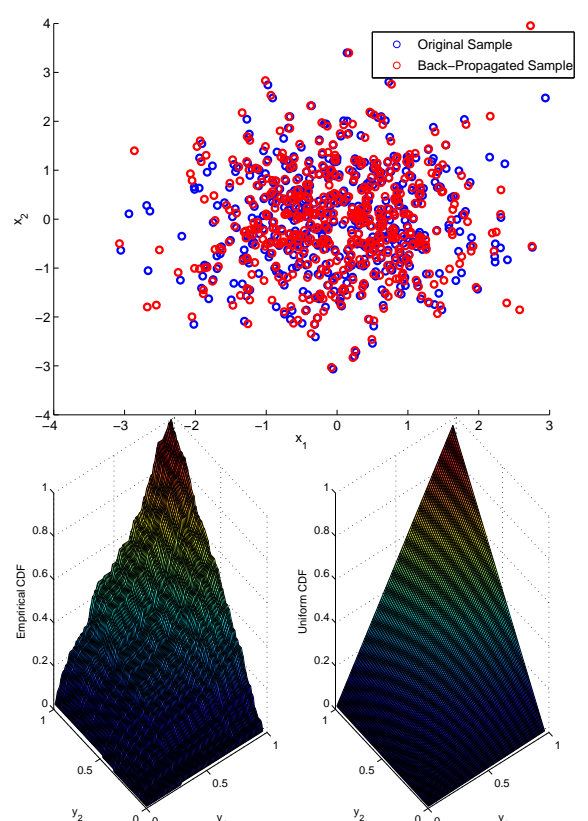

Fig. 2. (Top) Scatter plot of initial and the back-propagated elements for a given sample. Blue circles are the original sample and the red ones are the back-propagated sample. (Bottom) Plot of Empirical CDF of $\hat{y}_{0}^{j}$ (left) and uniform CDF (right) for a given sample

For the KS test, the value of $\alpha$ is fixed to 0.05 . Hence, using (15) and (16), we get $\frac{K_{\alpha}}{\sqrt{N}}=0.0607$. For the given sample, the value of $D_{N}$ was 0.0455 . Hence, the null hypothesis, that the sample is drawn from a standard normal distribution, is accepted with a significance level $\alpha=0.05$.

Figure (3) show plot for $D_{N}$ values for all the 100 samples drawn. The samples above red line fail the test if $\alpha=0.05$ and the samples above the black line fail, if $\alpha=0.01$. It is observed that only 28 samples fail the test when $\alpha=0.05$ and 14 samples fail when $\alpha=0.01$. It can be seen that, as the significance level $\alpha$, is decreased, the chances that the null hypothesis is accepted increases. As mentioned before in (17), the value of $D_{N}$ obtained after KS test is a random variable, whose CDF converges to Kolmogorov distribution exponentially. Figure (4) show plots for the empirical CDF of $D_{N}$, and the Kolmogorov $\mathrm{CDF}$ in $\mathbb{R}[0,1]$, for a fixed $N_{t}=21$. We vary the number of samples, $N_{s}$ and observe the convergence in $\sup _{x \in \mathbb{R}} \mid F_{N_{s}}(x)-$ $F_{K}(x) \mid$. The variation is plotted in fig.(5). It can be seen that $\sup _{x \in \mathbb{R}}\left|F_{N_{s}}(x)-F_{K}(x)\right|$ decreases exponentially as we increase $N_{s}$, which is in compliance with the theory. Due to

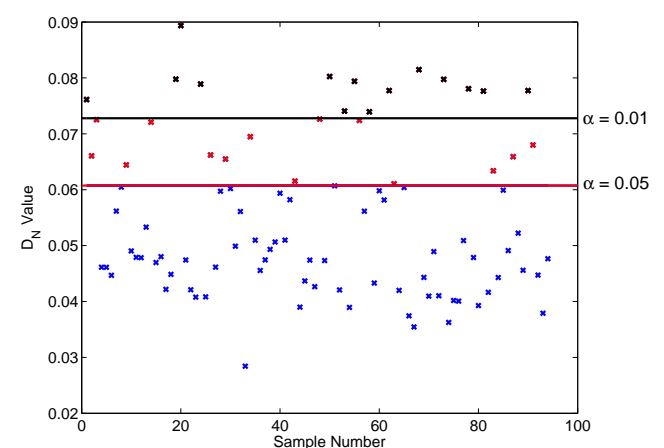

Fig. 3. $D_{N}$ value for all the 100 samples. The samples above the red and black lines fail when $\alpha=0.05$ and 0.01 respectively.

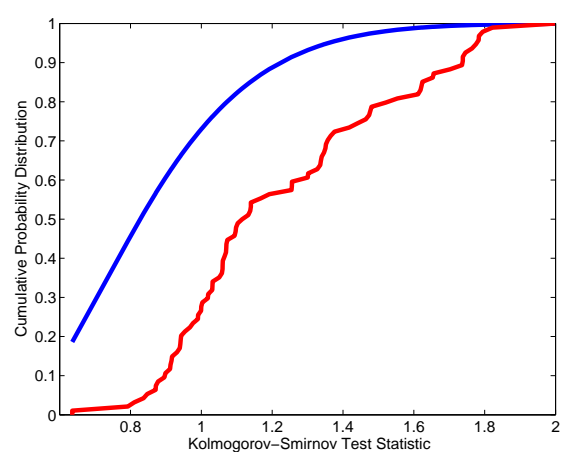

Fig. 4. Empirical CDF of $\sqrt{N} D_{N}$ (red) and Kolmogorov CDF (blue)

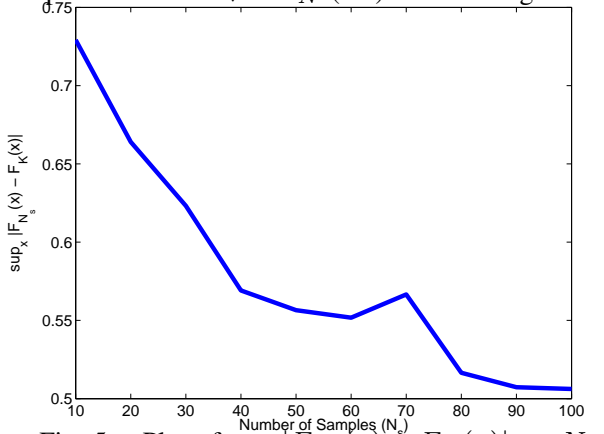

Fig. 5. Plot of $\left.\sup _{x \in \mathbb{R}} \mid F_{N_{s}}(x)\right)^{-} F_{K}(x) \mid$ vs. $N_{s}$

the fact that the convergence is in probability but only one realization of $\sup _{x \in \mathbb{R}}\left|F_{N_{s}}(x)-F_{K}(x)\right|$ has been plotted here, we do not observe a monotonic behavior. But, there are very few outliers, as the probability in the left hand side of (17) can be decreased arbitrarily by varying $\epsilon$.

\section{ApPliCATION to NONLINEAR Filtering}

We apply the proposed KLPF methodology to estimate states of a nonlinear system. The nonlinear estimation algorithm used is same as the one described in [9]. Here, we draw random particles (elements) from the domain $\mathbf{D}_{z}$ of $z(t=0)$. Propagation of uncertainty is done using (12). By using this framework for nonlinear estimation purposes, we get the exact value of prior PDF after propagation. This gives us significant advantage over particle filters where, generally the prior PDF is approximated using histograms. Bayesian inference is used to update the posterior PDF from prior for each particle.

In the present work, the system used is the Vanderpol's oscillator described by (13). We use a nonlinear measurement model given by, $y(t)=x_{1}^{2}(t)+x_{2}^{2}(t)+\eta(t)$, where $\eta(t)$ is zero mean Gaussian measurement noise with autocorrelation $R=6 \times 10^{-2}$. The process noise, $\xi(t)$ is also considered to be Gaussian with zero mean and an autocorrelation $Q=$ $6 \times 10^{-1}$.

We consider the initial state uncertainty to be normally distributed with $\mathbf{E}[z]=[0,0]^{T}$ and $\mathbf{E}\left[z^{2}\right]=\operatorname{diag}(1,1)$. It is assumed that the initial states of the actual system is $[0.25,0.25]$, with initial error in estimation being $[0.25,0.25]$. The measurement update interval was fixed to $0.1 s$ and final time $T$ was assumed to be $T=1 \mathrm{~s}$.

The performance of the KLPF-based filter is compared with the generic particle filter given in [24]. The sample size for each estimator is fixed to 5000 elements. The simulations 

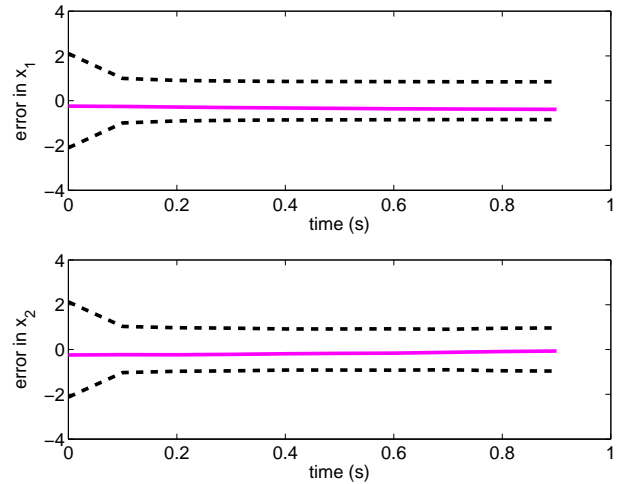

Fig. 6. KLPF-based filter. Magenta- error in estimation, black- $\pm 3 \sigma$ bounds
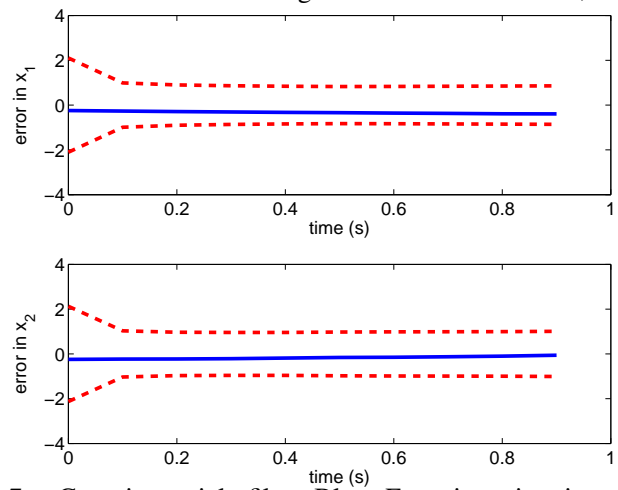

Fig. 7. Generic particle filter. Blue- Error in estimation, red- $\pm 3 \sigma$ bounds

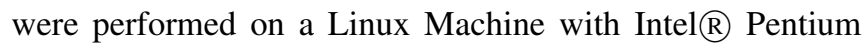
D processor. Figure (6) and Figure (7) show the $\pm 3 \sigma$ plots for the estimators. No major difference can be observed in the performance of the estimators as the errors in estimation are within the $\pm 3 \sigma$ limits and converge. But if we compare the computational time, KLPF-based estimator takes $58.46 \mathrm{~s}$ per filtering step, whereas the time taken by generic particle filter is $284.19 s$. Thus it can be concluded that, given same sample size and the same processing environment, KLPFbased estimator achieves similar performance as the generic particle filter, and is almost five times faster than the particle filter based estimator.

\section{CONCLUSIONS AND Future WORK}

In this work, an uncertainty propagation methodology is proposed for stochastic nonlinear dynamical systems, based on Karhunen-Loève (KL) expansion and Perron-Frobenius operator. The methodology uses KL expansion to approximate the process noise and Perron-Frobenius operator to predict the evolution of uncertainty. The method is illustrated by propagating uncertainty in a Duffing oscillator and a Vanderpol's oscillator. It was observed that the probability distribution of states, obtained after propagation, is in compliance with the underlying theory for these nonlinear systems. Moreover, the solution obtained with the approximated dynamics using KL expansion, was verified with the true solution, when applied to a Vanderpol's oscillator. It was found that the states of the approximated system converges in distribution to the true states of the system. A verification algorithm for the same has been presented here.

In future, we will focus on obtaining convergence relation between approximated dynamics and the true solution as a function of the number of terms in KL expansion.

\section{REFERENCES}

[1] R.E. Kalman. "A New Approach to Linear Filtering and Prediction Problems". Journal of Basic Engineering, Vol. 82, No. 1, 1960, pp $35-45$.

[2] M. Kumar, S. Chakravorty, and J.L. Junkins, "A Semianalytic Meshless Approach to the Transient Fokker-Planck Equation", Probabilistic Engineering Mechanics, Vol. 25, No. 3, 2010, pp. 323-331.

[3] R.A. Bellman, Dynamic Programming, Princeton University Press, NJ; 1957.

[4] A. Lasota, and M. Mackey, Chaos, Fractals and Noise: Stochastic Aspects of Dynamics, Springer-Verlag, NY; 1994.

[5] A. Halder, and R. Bhattacharya, "Dispersion Analysis in Hypersonic Flight During Planetary Entry Using Stochastic Liouville Equation", Journal of Guidance, Control and Dynamics, Vol. 34, No. 2, 2011, pp. $459-474$.

[6] L.C. Evans, Partial Differential Equations, Graduate Studies in Mathematics, Vol. 19, American Mathematical Society, Providence, RI; 1998.

[7] A. Halder, and R. Bhattacharya, "Performance Bounds for Dispersion Analysis: A Comparison Between Monte Carlo and Perron-Frobenius Operator Approach", Preprint, available at http://people.tamu.edu/ahalder/.

[8] A. Halder, and R. Bhattacharya, "Beyond Monte Carlo: A Computational Framework for Uncertainty Propagation in Planetary Entry, Descent and Landing", AIAA Guidance, Navigation and Control Conference, Toronto, ON, 2010.justel1997multivariate

[9] P. Dutta, and R. Bhattacharya, "Hypersonic State Estimation using Frobenius-Perron Operator", Journal of Guidance, Control and Dynamics, Vol. 34, No. 2, 2011, pp. 325-344.

[10] C. Soize, and R. Ghanem, "Physical Systems with Random Uncertainties: Chaos Representations with Arbitrary Probability Measure", SIAM Journal on Scientific Computing, Vol. 26, No. 2, 2004, pp. 395410.

[11] P. Spanos, and R. Ghanem, "Stochastic Finite Element Expansion for Random Media", Journal of Engineering Mechanics, Vol. 115, No. 5, 1989, pp. 1035-1053.

[12] D. Xiu, and J. Hesthaven, "High-Order Collocation Methods for Differential Equations with Random Inputs", SIAM Journal on Scientific Computing, Vol. 27, No. 3, 2005, pp. 1118-1139.

[13] B.J. Debusschere, H.N. Najm, P.P. Pébay, O.M. Knio, R. Ghanem, and O.P. Le Maitre, "Numerical Challenges in the Use of Polynomial Chaos Representations for Stochastic Processes", SIAM Journal on Scientific Computing, Vol. 26, No. 2, 2004, pp. 698-719.

[14] P. Langevin, "On the Theory of Brownian Motion", CR Acad. Sci.(Paris), Vol. 146, 1908, pp. 530-533.

[15] K. Karhunen, "Über lineare Methoden in der Wahrscheinlichkeitsrechnung", Ann. Acad. Sci. Fennicae. Ser. A. I. Math.-Phys, Vol. 37, 1947, pp. $1-79$.

[16] M. Loève, "Fonctions Aléatoires du Second Ordre", Processus Stochastic et Mouvement Brownien, supplement to P. Levy, Paris, Gauthier Villars, 1948.

[17] R. Ghanem, P. Spanos, Stochastic Finite Elements: A Spectral Approach, Springer-Verlag, NY; 1991.

[18] N. Cressie, Statistics for Spatial Data, Wiley-Interscience, NJ; 1993.

[19] M. Grigoriu, Stochastic Claculus: Applications in Science and Engineering, Birkhäuser, Boston; 2002.

[20] A. Leon-Garcia, Probability, Statistics and Random Processes for Electrical Engineering, Prentice Hall, NJ; 2008.

[21] I. Karatzas, and S. Shreve, Brownian Motion and Stochastic Calculus, Springer-Verlag, NY; 1991.

[22] A. Justel, and D. Peña, and R. Zamar, "A Multivariate KolmogorovSmirnov Test of Goodness of Fit", Statistics \& probability Letters, Vol. 35, No. 4, 1997, pp 251-259.

[23] A. Dvoretzky, and J. Kiefer, and J. Wolfowitz, "Asymptotic Minimax Character of the Sample Distribution Function and of the Classical Multinomial Estimator", Annals of Mathematical Statistics, Vol. 27, No. 3, 1956, pp 642-669.

[24] M.S. Arulampalam, and S. Maskell, and N. Gordon, and T. Clapp, and D. Sci, and T. Organ, and SA Adelaide, "A Tutorial on Particle Filters for Online Nonlinear/Non-Gaussian Bayesian Tracking". IEEE Transactions on Signal Processing, Vol. 50, No. 2, 2002, pp 174-188.

[25] G. Steinbrecher, and W.T. Shaw, "Quantile mechanics". European Journal of Applied Mathematics, Vol.19, No. 2, 2008, pp. 87-112. 